\section{Family relationships}

$\mathrm{T}$ he same company publishes both Nature and Nature Neuroscience. The journals share an editorial mission of providing high visibility for important scientific advances, as well as many specific policies, such as competing financial interest statements. Thus it is easy to understand why many authors find it difficult to believe that the journals' editorial processes are independent of one another. What do we mean by editorial independence? Most importantly, we mean that editors at one journal cannot access information about the papers under consideration at the other journal, unless the authors give permission for it to be shared.

The most common reason for authors to give such permission is that when Nature's editors reject a paper, in some cases they may suggest that the authors submit it to Nature Neuroscience. In most cases, these suggestions are based on the Nature editor's best judgment. When submitting such a paper to Nature Neuroscience, the authors must decide whether to continue the Nature review process, or to ask for a fresh start. Authors who want a fresh start are not required to tell us that the paper was submitted to Nature. In that case, we will not read the Nature reviews or know who wrote them (and it therefore remains possible that we will select some of the same referees by chance, in which case our policy is not to exclude them).

Authors who want to continue the Nature review process should provide a point-by-point response to the reviews with the submit- ted paper. Occasionally such papers are accepted without further review, or after informal consultation with one or more refereesfor instance if the Nature referees had no technical concerns but felt the paper was not suitable for Nature's broad audience. Other papers are rejected without further review, particularly if the authors have failed to address technical concerns raised by the Nature referees or if we judge the paper insufficiently novel or interesting for Nature Neuroscience. Often the revised paper is sent back to one or more Nature referees. We may not send the paper back to all the Nature referees, and may also choose to add a new referee at this point. However, we cannot honor requests such as "please do not send the paper back to referee 3," on the grounds that authors should not be permitted to selectively avoid negative viewpoints on their paper.

A key point is that authors may not change their minds later if the path they chose leads to rejection-either by providing Nature reviews that were not mentioned at the time of submission, or by asking for new referees if we decline the paper based on the Nature reviews or if the Nature referees do not recommend publication of the revised manuscript. Our ability to continue the Nature review process is intended as a service to authors, providing the opportunity to save time that might be lost by starting over at another journal. It is not intended as a guaranteed path to acceptance.

\title{
Changes at Nature Neuroscience
}

A lert readers may notice that the journal has a new look this month, including additional color and new fonts to improve readability. Most of the journal's contents will remain the same, although the section previously called Letters to the Editor will now be called Correspondence. In addition, we are inviting submissions in two new formats as part of our redesign.

First, we will begin publishing occasional Technical Reports, which will present primary research data on new techniques that are likely to be influential. This format is not a review of technology, but its primary report in the literature. We prefer that Technical Reports should include a new biological discovery to prove the usefulness of the technique, but this is not a requirement. Refinements of previously published techniques are not normally appropriate for Technical Reports.

We will continue to publish Reviews and Commentaries, but we will distinguish them from our second new format, Perspectives. Commentaries represent the personal viewpoint of their authors on issues of broad scientific interest, and should be written in an accessible, non-technical style. They may address questions of policy, science and society or purely scientific issues. Commentaries may or may not be peer reviewed, depending on their contents.
In contrast, Reviews and Perspectives are more formal, scholarly formats, which are always peer reviewed. A Review is an authoritative, balanced survey of recent developments in a research field. While authors may propose a specific viewpoint, controversies in the field must be treated even-handedly. The scope of a Review should be broad enough that it is not dominated by the work of a single laboratory, including the authors' own work. Publication as a Review implies that the editors believe the article represents as a balanced evaluation of the field.

Some of the more technical pieces that would formerly have been called Commentaries will now be published as Perspectives. This new format is intended for scholarly reviews and discussions of the primary research literature that do not meet the criteria for a Revieweither because the scope is too narrow, or because the authors are advocating a controversial position or a speculative hypothesis or discussing their own work. For example, two reviews advocating opposite sides in a research controversy would be published as Perspectives. We hope that this new format will allow us to bring more interesting ideas to the attention of our readers, and invite interested authors to send us a proposed outline by email to neurosci@natureny.com. 